\title{
OPTICAL FLOW APPLIED TO TIME-LAPSE IMAGE SERIES TO ESTIMATE GLACIER MOTION IN THE SOUTHERN PATAGONIA ICE FIELD
}

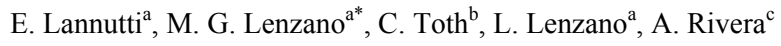 \\ ${ }^{a}$ Depto. de Geomática. IANIGLA-CCT, CONICET, Av. Ruiz Leal s/n. Mendoza, Argentina - (elannutti, mlenzano, \\ llenzano,)@mendozaconicet.gob.ar \\ ${ }^{\mathrm{b}}$ Department of Civil, Environmental and Geodetic Engineering, The Ohio State University, Ohio, USA - toth.2@osu.edu \\ ${ }^{\mathrm{c}}$ Centro de Estudios Científicos Chile, Valdivia, Chile - arivera@cecs.cl
}

Commission VI, WG VI/4

KEY WORDS: optical flow, time-lapse imagery, motion detection, glacier

\begin{abstract}
:
In this work, we assessed the feasibility of using optical flow to obtain the motion estimation of a glacier. In general, former investigations used to detect glacier changes involve solutions that require repeated observations which are many times based on extensive field work. Taking into account glaciers are usually located in geographically complex and hard to access areas, deploying time-lapse imaging sensors, optical flow may provide an efficient solution at good spatial and temporal resolution to describe mass motion. Several studies in computer vision and image processing community have used this method to detect large displacements. Therefore, we carried out a test of the proposed Large Displacement Optical Flow method at the Viedma Glacier, located at South Patagonia Icefield, Argentina. We collected monoscopic terrestrial time-lapse imagery, acquired by a calibrated camera at every 24 hour from April 2014 until April 2015. A filter based on temporal correlation and RGB color discretization between the images was applied to minimize errors related to changes in lighting, shadows, clouds and snow. This selection allowed discarding images that do not follow a sequence of similarity. Our results show a flow field in the direction of the glacier movement with acceleration in the terminus. We analyzed the errors between image pairs, and the matching generally appears to be adequate, although some areas show random gross errors related to the presence of changes in lighting. The proposed technique allowed the determination of glacier motion during one year, providing accurate and reliable motion data for subsequent analysis.
\end{abstract}

\section{INTRODUCTION}

Data acquisition methods on earth observation and monitoring applications, have seen great technological advancements in recent years, such as the introduction of airborne and terrestrial LiDAR (Light Detection and Ranging) and a wide variety of satellite sensors with varying geometric resolutions that can be combined with other techniques of data acquisition to substantially improve the quality of the observations (Paul et al., 2009). As a consequence, remote sensing techniques represent nowadays an attractive approach to study glaciers, where the main advantage lies in providing a more practical and economical alternative compared to field measurements.

Glacier changes have been surveyed with a variety of methods since the mid-nineteenth century. The optical images have been the most important types of imagery to monitor the glaciers because they provided different spatial resolutions for mapping glacier changes/dynamics, even at present. Aerial and terrestrial photogrammetry has become a widely used technique to map rough and complex glacier topographies. Thus, time-lapse imagery provides high temporal frequency and high spatial resolution over a long period of time, although it may be applied in reduced areas.

The study of glacier dynamics requires the detail mapping of surface velocities. Ice flow velocities vary along the glacier, following complex patterns defined by stress and strain rate distributions. In order to map these velocities, several methods have been used, including discrete surveys, and spatially distributed methods resulting in irregular or regular distribution grid points. In order to obtain a dense and accurate grid of ice velocities, a wide range of techniques of digital image processing have been proposed, including in the early 80 's, the
Image pyramids technique able to perform image blending (Szeliski, 2010). In parallel, coarse-to-fine strategies were also developed, such as intensity-based optical flow algorithms, among others.

The optical flow extracted from imagery is the result of the apparent movement pattern between objects, caused by either relative (deformation) or absolute movements. Estimating correspondence between pairs of points in two images remains one of the fundamental computational challenges in Computer Vision (Wedel et al., 2009). The objective of motion estimation is to compute an independent estimate of motion for each pixel, which is generally known as optical flow (Szeliski, 2010). The majority of today's methods strongly resemble the original formulation of Horn and Schunck (HS) (Horn and Schunck, 1981). In spite of the existence of a variety of optical flow techniques, the majority of the algorithms concern small displacements and only a few procedures have been developed to detect large displacements, such as those occurring with glaciers; note that large displacement is defined with respect to the image acquisition rate. The Large Displacement Optical Flow (LDOF) method (Brox et al., 2004, Brox and Malik, 2011) offers an interesting alternative to estimate large displacement between image sequences, and is based on a solid numerical method that includes a coarse-to-fine strategy using the socalled warping technique, and implements the non-linearized optical flow constraint for image registration. The constancy assumption of the gradient makes this method robust to grey values changes. Finally, the descriptor matching and the discrete optimizations provide subpixel accuracy.

Few studies of ice motion have been carried out using optical flow algorithm (Vogel et al., 2012; Bown, 2015). Thus, in this work, we propose the LDOF algorithm to estimate the motion 
of a glacier by terrestrial monoscopic time-lapse image series acquired by non-metric professional DSLR camera systems. The test was carried out at the Viedma glacier, Southern Patagonia Icefield (SPI), Argentina. This study aims to obtain efficient solutions at pixel level and determine ice velocities in the terminal part of the glacier. The outline of this paper is as follows: Section 2 provides a detailed description of the study area and data collection. Section 3 reviews the methodology proposed. Section 4 presents the results with analysis, and Section 5 provides the conclusion.

\section{TEST AND DATA ACQUISITION}

The South Patagonia Ice field (SPI) is located in South America, Argentina and Chile, covering an area of $13,000 \mathrm{~km}^{2}$ with an average length of approximately $30-40 \mathrm{~km}$ at a mean altitude of 1,191 m ASL (Aniya and others, 1996). Presently, after Antarctica and Greenland, it is the third largest reservoir of fresh water on continental shelves. The Viedma glacier is located at $49^{\circ} 31^{\prime}$ S, $72^{\circ} 59^{\prime}$ W, Parque Nacional Los Glaciares, South Patagonia Icefield, Santa Cruz, Argentina (Fig. 1). This is an important calving glacier in the region covering an area of $945 \mathrm{~km}^{2}$ (Aniya et al., 1996). The glacier was selected for this study due to the availability of several studies on the surface changes, carried out over the last 30 years (Skvarca et al., 1995, Aniya et al., 1996; Lopez et al., 2010; Rivero et al., 2013).
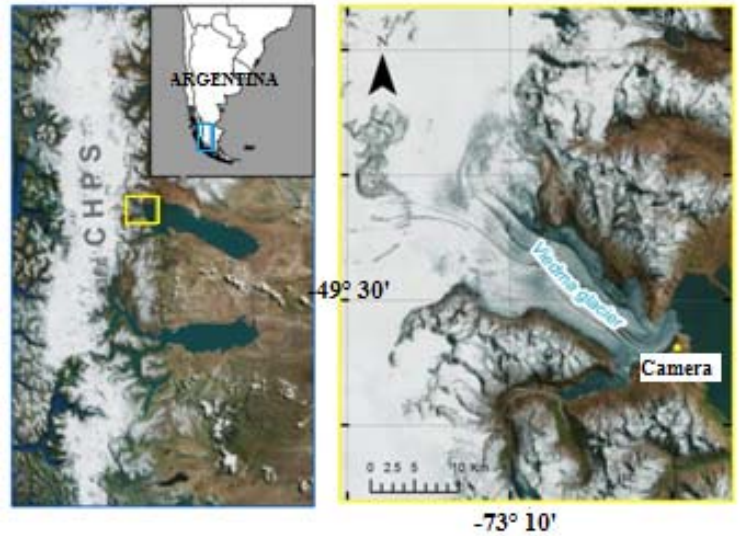

Figure 1. Map of the study area.

To support the field image acquisition, an integrated data acquisition system was built around the CANON EOS Mark II DSLR camera; pixel size: $7.2 \mu$, objective focal length: $50 \mathrm{~mm}$, and FOV: $46^{\circ}$. The camera was calibrated several times, initially by the United States Geological Survey (USGS), and prior to field deployment. The system is powered by one $12 \mathrm{~V} / 7 \mathrm{Ah}$ lead acid battery, charged by two $38 \mathrm{~W}$ solar panels. The camera with the supporting electronic systems is protected by a waterproof enclosure, with a viewing port and a visor to reduce reflections. An inspection port in the rear of the enclosure provides visual access to monitor status. The image acquisition system was installed on a rigid metal structure, fixed to outcrops of the South margin of Viedma Glacier. The location provides a good side view of the curve of Viedma Glacier see Fig. 2. The image acquisition started on April 17, 2014 , and one picture was captured at $12 \mathrm{pm}$ local time every day until April, 82015 (total of 356 images).

\section{PROPOSED APPROACH}

The overall processing workflow is shown in Fig. 3. Except for the image resizing, all the subsequent processes were made in
Matlab. In order to lower computational costs, the original sizes of the images were reduced based on the Region of Interest (ROI), the glacier area, Fig. 4 a and b show the elimination of the sky and mountain range at the top of the photo. Note that, changing objects, such as clouds and snow may affect the movement detection in the LDOF algorithm. Next, the Motion Detection Processes (MDP) method is executed, including the Correlated Image Filter (CIF) process and the LDOF computation that provides the uncertainty estimation.

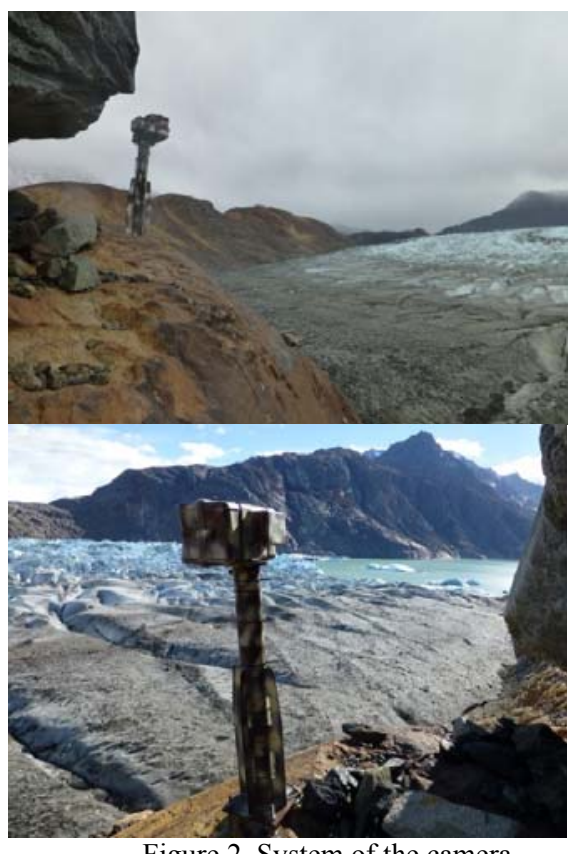

Figure 2. System of the camera.

\subsection{Correlated Image Filter (CIF)}

We implemented the CIF processes with the assumptions that optical flow brightness is constant over time, and nearby points in the image move in a similar way (Schalkoff, 1989). Thus, important changes in lighting should be avoided to assume that changes of image irradiance are only caused by the image sequence (Klette, 2014). This generally involves the summation of color differences between corresponding pixels over the image. In our study case, the daily changes in solar radiation and the seasonal snow cover were corrected by the CIF.

First, at pair of possible images are selected, $\mathrm{IM}_{n}$ (Master) and $I_{n+1}$ (Slave), both of which are separated into the three RGB bands in order to perform a correlation for each channel separately for both images. The mean correlation value of each of the three channels is analyzed. If the mean value is equal or greater than 0.94, then the pair remains selected. Otherwise, the initial threshold of 0.94 is iteratively reduced in 0.005 steps and a new correlation between the $\mathrm{IM}_{n}$ and the $\mathrm{IS}_{n+2}$ is computed until the pair of images surpasses the descending correlation threshold. The reason to apply a variable correlation threshold is to avoid correlation between images that are too separated in time, as the objects may change substantially due to the glacier's movement. This sequence is repeated $n$ times for all the images, where $\mathrm{IS}_{n+m}$ becomes $\mathrm{IM}_{n+m}$, making the correlation between the latter and the $\mathrm{IS}_{n+m+1}$. As a result of this process, correlated pairs of images are obtained throughout the course of the entire sequence, where the changes in luminosity are minimized, and each selected pair is ready for the LDOF to be calculated. 


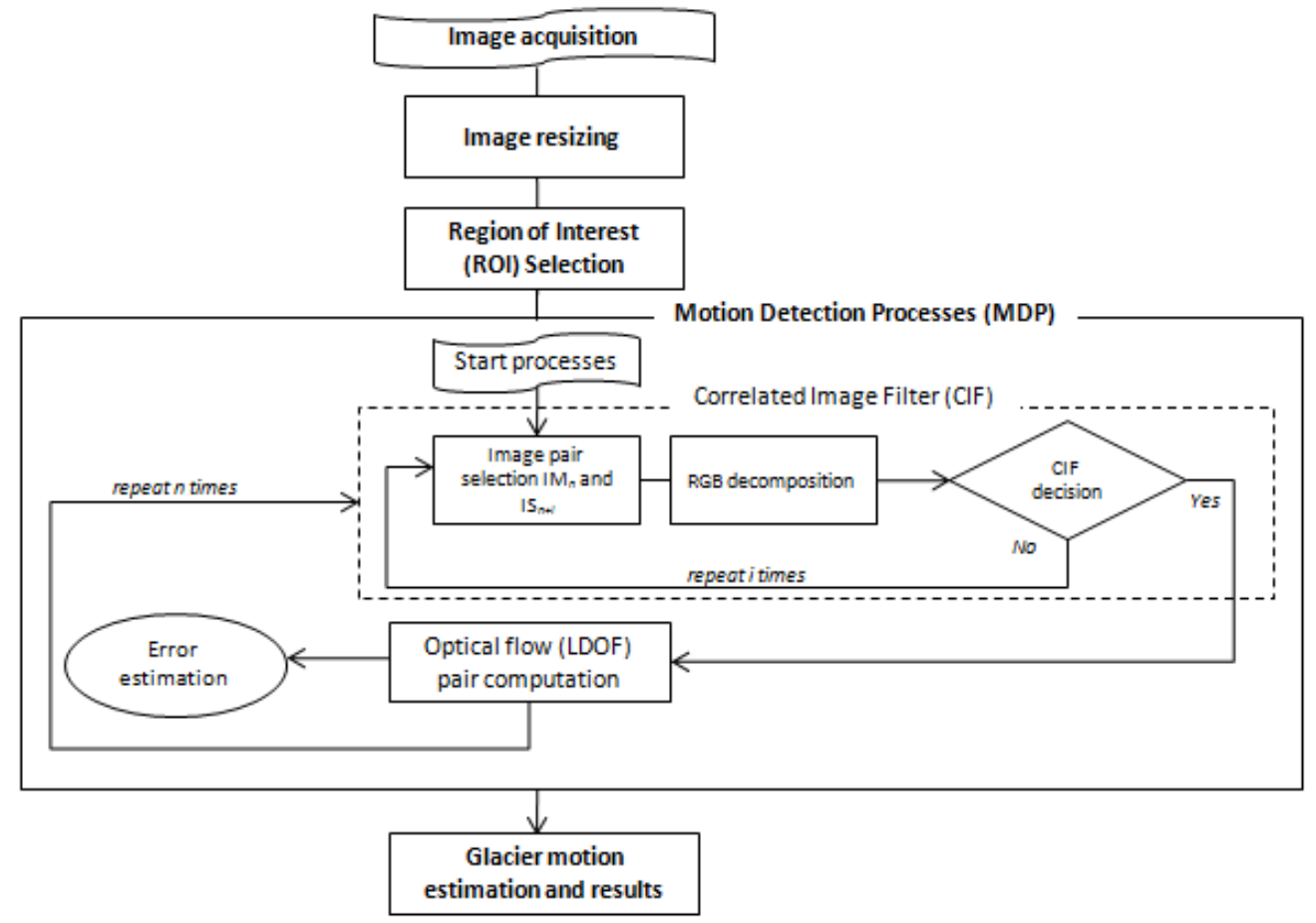

Figure 3. Workflow of the present study.

Figure 5 shows the result of the CIF. The blue line describes the correlation value for the mean value of the three RGB bands for the $n$ pair of resulting images from each search. The black line corresponds to the threshold of the iterative correlation test that decreases until it coincides with the RGB correlation value and hence passes the test. The red points show the 127 selected images by the CIF, i.e., $36 \%$ of the images passed the test. The lower correlation value for the test was 0.85 , and corresponds to the period between images 40 and 60 , coinciding with a major snow storm that lasted 20 days.

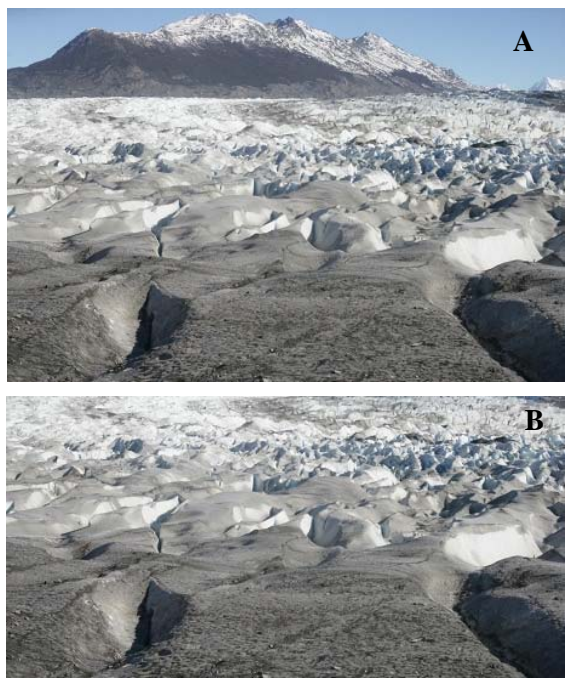

Figure 4. Original and ROI reduced image.

\subsection{Optical Flow (LDOF)}

We use the LDOF Matlab implementation developed by Brox et al. (2004), Brox and Malik (2011). After having done tests with other optical flow algorithms, the LDOF was the one that produced the best results in the motion estimation. This algorithm implements a coarse-to-fine variational framework between two images $I_{1}$ and $I_{2}$, and computes the displacement field $w(x)=(u, v)$ by minimizing the functional energy $E(w)$ using the following model (Eq. 1):

$$
\begin{gathered}
E(w)=E_{\text {color }}(w)+\gamma E_{\text {gradient }}(w)+\alpha E_{\text {smooth }}(w)+ \\
\beta E_{\text {match }}\left(w, w_{1}\right)+E_{\text {desc }}\left(w_{1}\right)
\end{gathered}
$$

Where $\alpha, \beta$ and $\gamma$ and are tuning parameters which can be determined manually according to qualitative evidence on a large variety of videos, or can be estimated automatically from ground truth data. $w_{1}$ is an auxiliary variable (Brox and Malik, 2011).

Equation 1, involves in the first and second terms the common assumption that corresponding points should have the same gray value or color and gradient constancy. The third term emphasizes the strength and importance of regularity constraints in optical flow estimation by a robust smoothness. The last two terms combine descriptor matching with the variational model and its coarse-to-fine optimization. The descriptor matching method is based on densely computed Histogram of Oriented Gradients (HOG). Each gradient histogram comprises 15 different orientations and is computed in a $7 \times 7$ neighborhood. The method with changing resolutions is performed by dividing the original problem into a sequence of sub-problems at different levels of resolution. Using image pyramid, the transitions between levels to resolve the Euler-Lagrange equations by iterations by smoothing. 
In the first place, results were evaluated in a qualitative manner, where the color-coded flow field has a meaning associated with the physical variable of study, which, in this case, is the movement of the glacier. In accordance with Streinbruecker et al. (2009), we checked the consistency of the flow-field by reconstructing the first of the two frames using the second frame and the estimated motion field $w$ according to (Eq. 2):

$$
I_{1}^{r}(x)=I_{2}(x+w(x))
$$

If the resultant flow is adequate, then the reconstruction of $I_{1}^{r}$ has to be identical to the $I_{1}$. In order to estimate the error, we calculated the absolute difference between the pair of images to estimate the final error by computing the mean of each pixel, throughout the whole sequence.

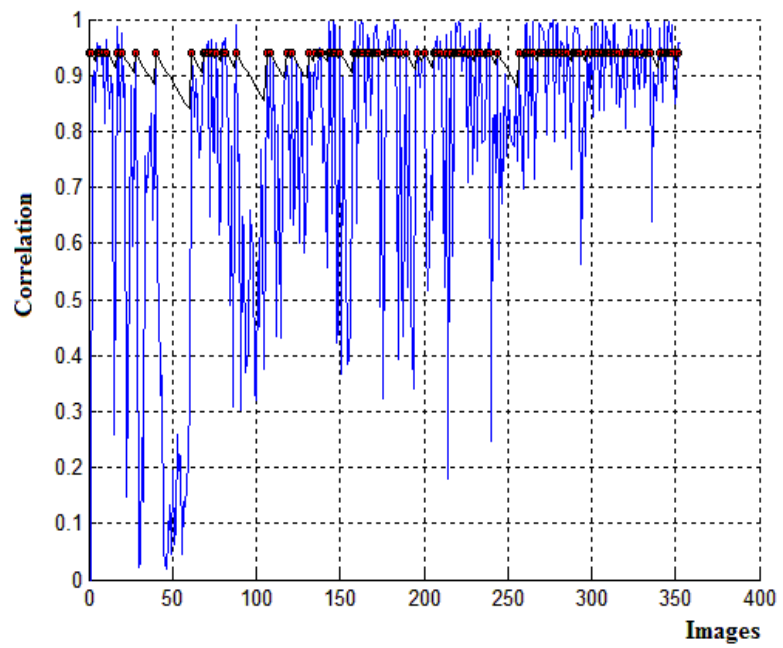

\section{RESULTS: MOTION DETECTION PROCESSES}

In general, ice flow velocities on a valley glacier cross section have maximum values at the centre of the valley and go down to a minimum at the margins. Along the valley, ice velocities magnitudes varies longitudinally depending on several factors, such as surface slope, mass balance distribution and, for the main aim of this paper, on the glacier front conditions. On calving glaciers, the velocities can reach a maximum at the glacier front, due to the pulling effect of high calving rates. These high rates can be enhanced when near buoyancy conditions at the front are reached by a glacier calving into deep waters (Rivera et al, 2012). Very little is known about the ice flow velocities near the front of Viedma glacier, and about the ice-lake interactions taking place in this place. In order to better understand the velocity field at the lower end of the glacier, ice velocities were obtained by means of the vector field flow based upon 354 photographs obtained from a fixed camera installed near Viedma glacier ice front. Figure 6 shows the resulting velocities pictured in red for the fast flow) area, mainly concentrated at the central part, and in blue for the slowly moving ice areas, mainly located toward the margins. This pattern is very consistent with the expected ice flow acceleration toward the glacier front central part, and with slow moving ice near the margins.

This near front acceleration has been previously described for other calving glaciers in Patagonia (Sakakibara et al., 2014), where calving is driven by water depths near the front. In Viedma glacier, this is confirmed by the recently surveyed bathymetry of the lake, where up to $571 \mathrm{~m}$ water depths were detected. Note that, since April 2014 to March 2016 the central part of the glacier front retreated near $800 \mathrm{~m}$ as detected by comparing satellite images.

Figure 5. Results of CIF processes, 127 images were selected.
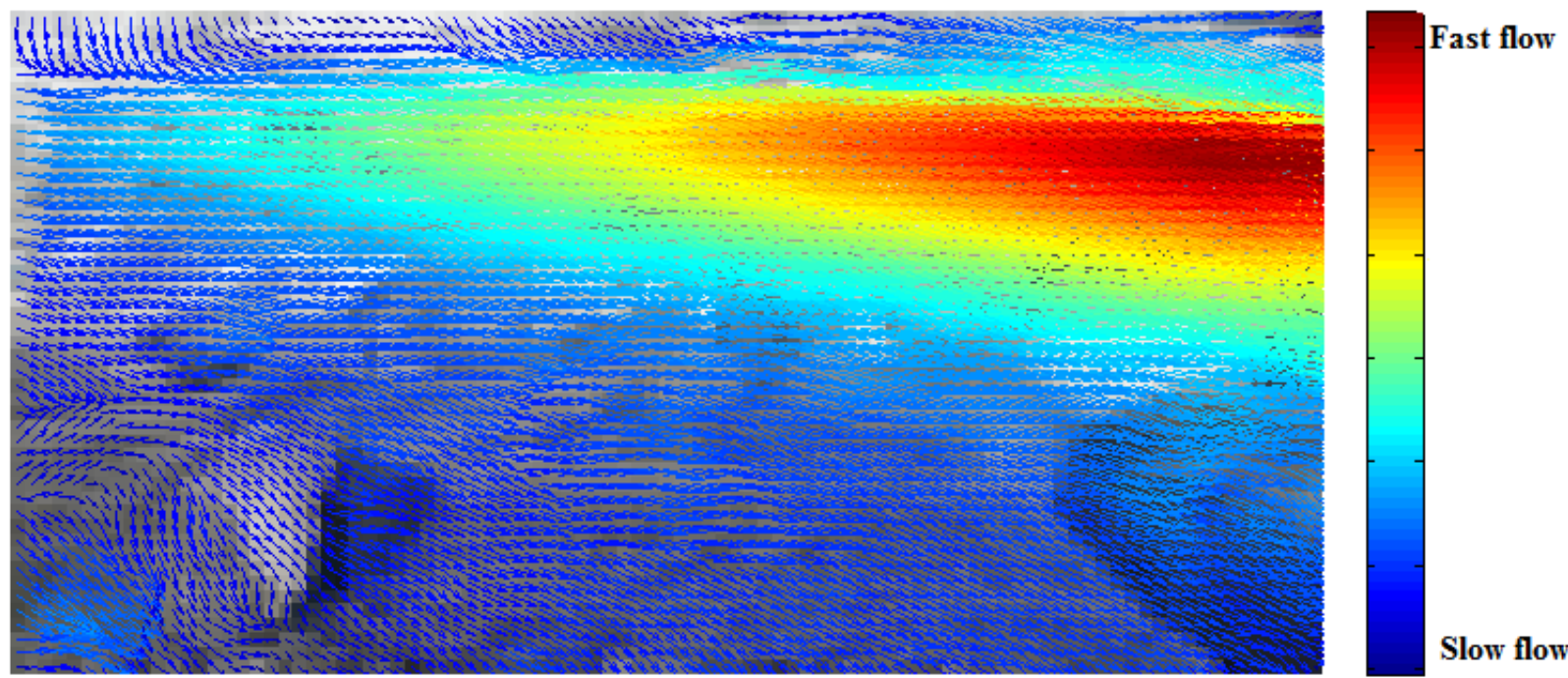

Figure 6 . Color flow vectors, their magnitude are blue=slow flow and red=fast flow. 
Figure 7 shows the mean error (blue) and the standard deviation (black) in each image pair and to the entire time-lapse sequence, where the mean error is $3.7 \pm 5.7$ in pixels. The biggest errors correspond to periods of large lighting changes, such as shown in the 10,21 and 24 pairs. These changes are related to the presence of snow cover and no uniform melting on the glacier surface as mentioned Vogel et al. (2012).

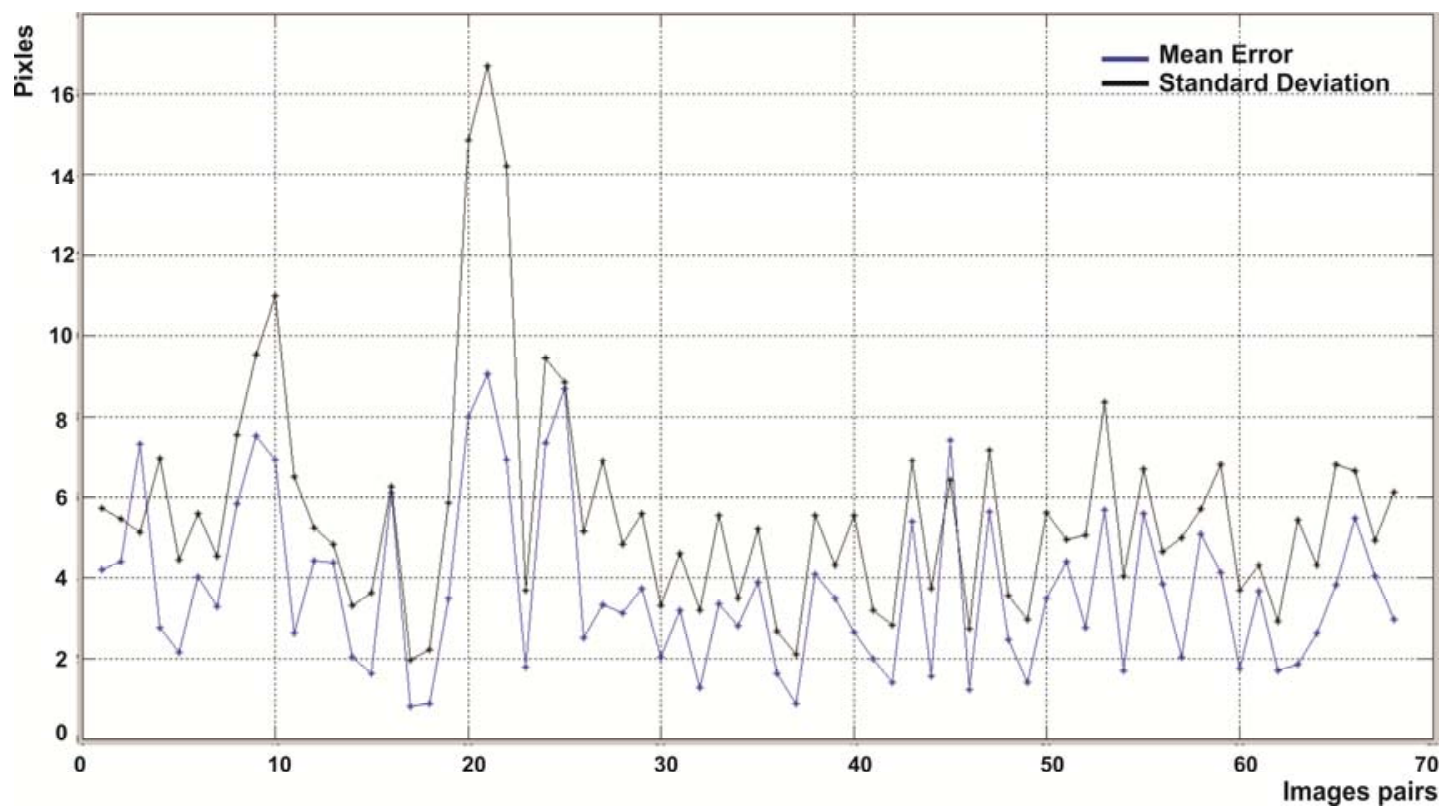

Figure 7. Mean Error and Standard deviation distribution for the images pairs processed by LDOF.

Figures $8 \mathrm{~A}$ and $\mathrm{B}$ show the reconstructed errors for two examples. The top frames show the photos that define the image pair for the LDOF is computed. Figure 8A shows an example of the error in the case of changing lighting conditions, resulting in significant errors in the computation of LDOF. Below the left image, the results of the LDOF are shown with color-coding for better visualization (Liu et al. 2010). Each pixel symbolizes a vector where the magnitude and orientation are function of the tonality and saturation of the pixel value. Below the right image, the error reconstruction is shown. The biggest errors are related to the presence of clouds, such as in the lower area that can be associated to shadows in the crevasses of the glacier, and the presence of changes in snow cover. In contrast, Figure $8 \mathrm{~B}$ shows an example with good lighting conditions where the error remained low over the entire area. Note the interesting situation in the middle of the frame, where the presence of people is observed, clearly indicating the ability of the LDOF to detect small changes with high precision.

In the image pair computations, yielding significant errors, we were able to easily identify the situation due to the fact that the movement of the glacier is lower than the motion recorded by the disappearance of the objects between the images that form the pair. Thus, we have demonstrated that the proposed method with respect to changes in brightness between the images, good results with small errors can be obtained.

\section{CONCLUSIONS}

In this work, we have applied dense optical flow field methods to estimate with high precision glacier movement. The proposed methods of MDP integrated by CIF and LDOF algorithms worked very well in the detection of the movement of Viedma glacier. The used approach starts with the ROIs selection aiming to decrease the computational time, and improving the results by eliminating zones that may only introduced noise. In natural environments, such as glaciers, there are a continuously changing lighting due to the presence of clouds, snow cover, and objects that appear or disappear, resulting in variations of radiometric conditions (brightness) in the photos affecting all the subsequent processes. Thus, the developed CIF proved to be essential to the LDOF algorithm. Thanks to this, the optimization and improvement of the brightness changes resulted in a better performance. Though only $36 \%$ of the images passed the applied test, they were enough for estimating the motion of Viedma glacier.

In our tests, the combination of terrestrial time-lapse data collection and the efficient LDOF solution at sub-pixel level accuracy provided a useful solution to detect the large daily changes. The estimation error was evaluated qualitatively and quantitatively, where the error reconstruction was computed and yielded a mean value of $3.7 \pm 5.7$ pixel. These values are related to the ability of LDOF to detect small structures changes indicating ice motion. Although there are few examples of optical flow algorithms applied for detecting ice velocities, we have been able to demonstrate the capabilities of LDOF for this purpose. The resulting velocities are consistent with the expected ice flow in a calving glacier with high velocities near the ice front.

Future work will focus on studying the relationship between the three-dimensional motion of objects and observers and the characteristics of the optical flow field. It will be relevant to demonstrate the important factors influencing changes in optical flow over the image from a not planar surface. This technique will help to better quantify the glacier dynamics. 

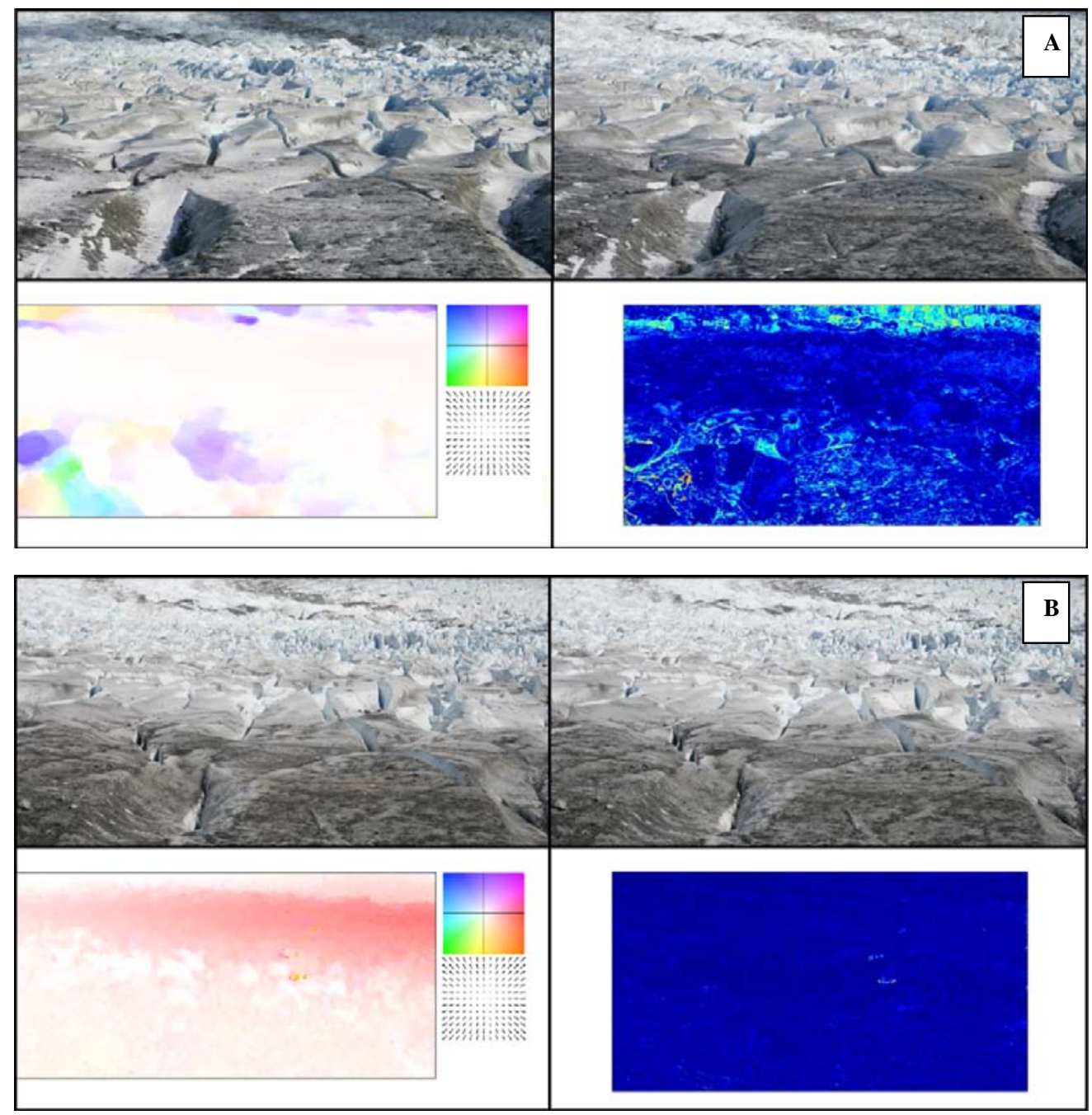

Figure 8. The top frames show the image pair selected to compute the LDOF. Left down shows the flow field estimated with colorcoding for a better visualization. Right down show the error reconstruction for the pair. A. An example of changing lighting conditions, and the mismatch of the glacier motion is denoted. B. An example with good lighting conditions, although the glacier has a large displacement is correctly matched.

\section{ACKNOWLEDGEMENTS}

The authors would like to thank Robert Bruce for his help in the English version, and Adalberto Ferlito, Simon Key and Andrés Lo Vecchio for field assistance. Parque Nacional Los Glaciares provided support in the area. Fieldwork was funded by grant PICT 2921-2012 and PICT 1995-2013. Agencia Nacional de Ciencia y Tecnología Argentina (ANCyT).

\section{REFERENCES}

Aniya, M., Sato, H., Naruse, R., Skvarca, P., and Casassa, G., 1996. The use of satellite and airborne imagery to inventory outlet glaciers of the Southern Patagonia Icefield, South American Photogrammetry Engineering Remote Sensing, 62(12), pp. 1361-1369.
Bown, F. (2015). Dinámica frontal de un glaciar de descarga oceánica: Glaciar Jorge Montt, Campo de Hielo Patagónico Sur. Mg. Thesis. Universidad de Concepción, Chile. pp 104.

Brox, T., Bruhn, A., Papenberg, N., and Weickert J., 2004, High Accuracy Optical Flow Estimation Based on a Theory for Warping. In: Proceedings 8th European Conference on Computer Vision, Springer LNCS 3024, T. Pajdla and J. Matas (Eds.), Prague, Czech Republic, Vol. 4, pp. 25-36.

Brox, T. and Malik, J., 2011. Large Displacement Optical Flow: Descriptor Matching in Variational Motion Estimation. IEEE Transactions on Pattern Analysis and Machine Intelligence. 33(3), pp. 500-513.

Horn, B. and Schunck, B., 1981, Determining optical flow. Artificial Intelligence, 17, pp. 185-203.

Klette, R., 2014. Concise Computer vision. An Introduction into Theory and Algorithms. Springer. New Zeland. pp.429. 
Liu, C. and Yuen, J., 2010, SIFT Flow: Dense Correspondence across Scenes and its Applications, Pattern Analysis and Machine Intelligence. IEEE Transactions On 33.5 (2011), pp. 978-994.

Lopez, P., Chevallier, P., Favier, V., Pouyaud, B., Ordenes, F., and Oerlemans, J., 2010, A regional view of fluctuations in glacier length in southern South America, Global Planetary Change, 71(1-2), pp. 85-108.

Paul, F., Kääb, A., Rott, H., Shepherd, A., Strozzi, T. and Volden, E., 2009, GlobGlacier: A new ESA project to map the World's glaciers and ice caps from space, European Remote Sensing Laboratories (EARSe)L eProceedings 8.

Rivera, A., Koppes, M., Bravo, C., and Aravena, J.C., 2012, Little Ice Age advance and retreat of Glaciar Jorge Montt, Chilean Patagonia, Climate of the Past, 8, pp. 403-414.

Riveros, N., Euillades, L., Euillades, P., Moreiras, S., and Balbarani, S., 2013, Offset tracking procedure applied to high resolution SAR data on Discussions Viedma Glacier, Patagonian Andes, Argentina, Advances in Geosciences, 35, pp. $7-13$.

Sakakibara, D. and Sugiyama, S., 2014, Ice-front variations and speed changes of calving glaciers in the Southern Patagonia Icefield from 1984 to 2011. Journal of Geophysical Research: Earth Surface, 119, doi: 10.1002/2014JF003148.

Schalkoff, R. J., 1989, Digital Image Processing and Computer Vision: An Introduction to Theory and Implementations, Wiley, New York.

Skvarca, P., Rott, H., and Stuefer, M., 1995, Synergy of ERS-1 SAR, XSAR, Landsat TM imagery and aerial photography for glaciological studies of Viedma Glacier, southern Patagonia, In: Proceedings, VII Simposio Latinoamericano de Percepcion Remota, SELPER, Puerto Vallarta, Mexico, pp. 674-682,

Steinbrücker, F., Pock, T. and Cremers, D., 2009, Large displacement optical flow computation without warping. In: IEEE International Conference on Computer Vision, pp. $1609-1614$

Szeliski, R., 2010, Computer Vision: Algorithms and Applications. Springer, pp. 957.

Vogel, C., Bauder, A. and Schindler, K., 2012, Optical flow for glacier motion estimation, In: 22 nd ISPRS Congress, Melbourne, Australia.

Wedel, A., Cremers, D., Pock, T., and Bischof, H, 2009, Structureand motion-adaptive regularization for high accuracy optic flow. In: Proceedings of the IEEE international conference on computer vision. 